\title{
Cuotas de contaminación y tecnología medioambiental diferenciada en presencia de inversión extranjera directa
}

\author{
Pollution quotas and differentiated environmental technology in the \\ presence of foreign direct investment
}

\begin{abstract}
Salvador Sandoval Bravo*, Rafael Salvador Espinosa Ramírez
Centro Universitario de Ciencias Económico Administrativas, Universidad de Guadalajara, México
\end{abstract}

Recibido el 25 de julio de 2019; aceptado el 28 de febrero de 2020

Disponible en Internet el: 3 de marzo de 2020

\section{Resumen}

El propósito de este trabajo, a partir de un modelo teórico, es determinar las políticas ambientales que maximicen el bienestar de un país receptor de inversión extranjera directa. En un mercado de un bien homogéneo las empresas foráneas compiten contra empresas nacionales bajo condiciones oligopolísticas. Se utiliza como instrumento de control la cuota de contaminación diferenciada, y se asume que las empresas de la inversión extranjera directa poseen tecnología más eficiente para abatir la contaminación. Se concluye que si la desutilidad por contaminar es considerablemente elevada, el gobierno del país anfitrión impone una cuota de contaminación cero tanto a las empresas locales como extranjeras; pero en el caso que la desutilidad por contaminar no sea muy alta, el gobierno permite a estas firmas cierta cantidad de emisiones dependiendo de la eficiencia relativa de cada tipo de empresa.

Código JEL: Q52, F18, F21

Palabras clave: Cuotas de contaminación; Políticas medioambientales

\footnotetext{
*Autor para correspondencia

Correo electrónico: salvsanb@cucea.udg.mx (S. Sandoval Bravo).

La revisión por pares es responsabilidad de la Universidad Nacional Autónoma de México. 


\begin{abstract}
The objective of this document, based on a theoretical model, is to determine optimal differentiated pollution quotas established by a country receiving foreign direct investment. Foreign firms compete against national firms for a homogeneous product in an oligopolistic market. It is assumed that foreign firms are more efficient in abating pollution than national firms. It is concluded that, if the disutility produced by pollution is considerably high, the domestic country government imposes a zero pollution quota on both local and foreign companies. But in the event that the disutility is sufficiently low, the domestic government allows both firms a certain amount of emissions depending on the relative efficiency of each type of company.
\end{abstract}

JEL code: Q52, F18, F21

Keywords: Pollution quotas; Environmental policies

\title{
Introducción
}

La inversión extranjera directa (IED) se ha considerado una fuente de progreso para muchas economías en desarrollo. Se argumenta que la IED promueve externalidades positivas en las economías en desarrollo como efectos tecnológicos colaterales, empleo, oportunidades financieras, entre otros. Así, las empresas extranjeras pueden promover el acceso a nuevas tecnologías, la formación de capital, la estimulación de la industria local, un bajo precio de los productos básicos, nuevos empleos y la reducción de la degradación ambiental, ya que, generalmente estas empresas poseen una tecnología más eficiente. Por lo tanto, en los países en desarrollo, la entrada de IED es crucial para su desarrollo. De acuerdo a la United Nations Conference on Trade and Development (2017), en 1995, el flujo global de IED fue de 341,137 millones de dólares; en 2005 alcanzó 948,933 millones de dólares, para llegar a su máximo histórico en el 2015 con 1,921,306 millones de dólares. Esa misma tendencia se cumple en el flujo de IED hacia los países en vías de desarrollo, que recibieron 117,767; 331,468 y 744,032 en los mismos períodos, lo que representa el 33\%, 34\% y 39\% de los flujos globales de IED.

Existe una amplia literatura referente a la IED, como Caves (2009); Cantwell (1994); Borensztein, Gregorio y Lee (1998); y Markusen y Venables (1999); por mencionar algunos. En estos trabajos se manifiesta que la IED no garantiza, necesariamente el desarrollo de los países. Además, se supone que la IED interactúa con la inversión nacional y considera específicamente la cuestión del medio ambiente. De hecho, una de las características básicas de la IED del modelo desarrollado es que estas empresas son tecnológicamente más eficientes para reducir la contaminación. 
Por otro lado, de acuerdo a la United Nations Environment Programme (2018), el uso cada vez más intensivo de recursos naturales en los procesos productivos ocasiona mayor contaminación (debida principalmente a la emisión de grandes cantidades de $\mathrm{CO}_{2}$ en la atmósfera), cuyos efectos son cada vez más devastadores y costosos. En este sentido, las consecuencias adversas se manifiestan, tanto en la salud humana al incrementarse los índices de enfermedades respiratorias, intestinales y auditivas; como en el medioambiente por el incremento acelerado del calentamiento global y el efecto de invernadero, que a su vez aumenta la incidencia e intensidad de tormentas, huracanes y tornados, provocando además, la alteración de ciertos ecosistemas y la posible extinción de especies. Sin embargo, los gobiernos se muestran cautelosos de aplicar medidas extremas para reducir la contaminación, ya que dichas políticas pueden aumentar considerablemente los costos industriales y reducir considerablemente la competitividad internacional de las empresas.

En lo que se refiere a la entrada de IED y al factor medioambiental, en el aspecto microeconómico (es decir, a nivel de las empresas o sector productivo), que es el enfoque de interés para este estudio, la literatura se divide generalmente en dos posturas: la hipótesis de los paraísos de contaminación y la hipótesis de los halos de contaminación. ${ }^{1}$

En lo que se refiere a la hipótesis de los paraísos de contaminación, según la cual las empresas multinacionales escogen preferentemente como destino de sus inversiones a países cuyos controles ambientales son menos estrictos, lo cual se traduce en la reducción de sus costos de producción (Eskeland y Harrison, 2003; Kanbur, Keen y Wijnbergen, 1995 y Chilchinisky, 1994). De esta forma, los países que cuenten con controles laxos a la contaminación se especializarán en tecnologías sucias y aquellos con que tengan controles más estrictos se especializarán en tecnologías limpias. En este sentido, Xing y Kolstad (2002) realizaron un estudio econométrico utilizando una muestra de 20 países en diferentes industrias y encontraron que para el sector químico existen indicios de que los controles ambientales no tan estrictos, atraen efectivamente inversión extranjera directa. En este sentido, Andonova (2003), estudia las políticas medioambientales en las economías de Europa del este en su proceso de orientación y conversión hacia una economía de mercado, citando casos de empresas altamente contaminantes que se han reubicado en estos países y operan bajo los estándares mínimos permisibles de control ambiental, muy por debajo de los implementados en plantas de sus países de origen. Queiroz (2018) analiza la conducta y del desempeño ambiental de empresas extranjeras de celulosa, localizadas en Argentina, Brasil, Chile y Uruguay; sus resultados confirman que, efectivamente existe en la región un proceso inminente de paraísos de contaminación, en razón de la instalación de tantas plantas industriales extranjeras de gran tamaño. Aun así, la existencia de paraísos de contaminación es muy debatible; pues no

\footnotetext{
${ }^{1}$ Véase Silva y Madeira (2015) para un estudio detallado del análisis de la literatura referente las relaciones existentes entre IED y medioambiente desde los enfoques microeconómico, macroeconómico y político.
} 
existe evidencia empírica suficiente de que las empresas multinacionales prefieran invertir e instalarse en países que permitan altos niveles de contaminación.

Por otro lado, algunos autores como Gentry (1998); Zarsky (1997); y Antweiler, Copeland y Taylor (2001); sugieren que las empresas multinacionales coadyuvan a mejorar el medioambiente de los países receptores de la IED, la denominada hipótesis de los halos de contaminación, ya que la tecnología de estas empresas, en general supera a la de los países locales, que suele ser más obsoleta. Existen estudios empíricos que apoyan esta hipótesis. Motta (2006), evalúa la industria brasileña y concluye que las multinacionales extranjeras generalmente adoptan más y mejores controles ambientales. Almeida y Rocha (2009) analizaron una muestra de empresas de las industrias de papel y celulosa, y petroquímica en Brasil, concluyendo que las grandes empresas extranjeras son líderes en lo que se refiere a control gerencial ambiental. Cole, Elliot y Strobl (2008) realizaron un estudio econométrico para empresas manufactureras extranjeras en Ghana, cuyos resultados sugieren que el mayor nivel de capacitación, experiencia gerencial y capacidad técnica se traducen en un mejor desempeño ambiental de las empresas multinacionales extranjeras. Haibo, Ayamba, Agyemang, Afriyie y Anaba (2019), analizan el efecto de la inversión extranjera directa sobre el desarrollo económico y sostenibilidad ambiental en China, concluyen que en general, este ha sido positivo, ya que la instalación de empresas extranjeras en los diferentes sectores, ha propiciado la adhesión de China a mejores políticas y prácticas mundiales de control de emisiones, ha recibido asistencia internacional para atender sus desafíos ambientales y ha favorecido la creación de un marco jurídico sólido que regula la contaminación emitida por las empresas, no obstante la regulación sobre las emisiones de $\mathrm{CO}_{2}$ siguen siendo laxas. Li, Dong, Huang y Failler (2019) llevaron a cabo un estudio econométrico basado en una muestra de 40 países que incluyeron variables de rendimiento ambiental, concluyeron que para países desarrollados la IED tiene un efecto positivo y significativo en el rendimiento ambiental sobre el país recepto de la IED, mientras que para países en vías de desarrollo también tenía un efecto positivo, pero marginal.

En lo que se refiere a los modelos teóricos que relacionen IED y la implementación de políticas ambientales la literatura es más bien escasa, a pesar de la relevancia que han adquirido en la actualidad. En este sentido, el presente trabajo desarrolla un modelo de equilibrio parcial para un país receptor de la IED en el mercado de un bien homogéneo, donde las empresas compiten bajo un esquema oligopólico. Se asume también que tanto las empresas nacionales como las extranjeras poseen tecnología para reducir la contaminación, pero considerando que las firmas de la IED poseen tecnología más eficiente para abatir dicha contaminación. El gobierno en el país anfitrión debe considerar el bienestar de todas las empresas locales y extranjeras, los consumidores y los costos sociales de la contaminación. Para regular las emisiones de las empresas, el gobierno utiliza como un instrumento de control ambiental, las 
cuotas de contaminación que son límites cuantitativos a la cantidad de emisiones aplicados a las empresas (Cropper y Oates, 1992). En este sentido las empresas deben asumir los costos de reducir sus emisiones mediante tecnología apropiada para ajustarse a la cantidad de emisiones determinada por el gobierno (Kolstad, 2012).

Entre los pocos estudios similares al desarrollado en este trabajo se pueden mencionar a Palomera y Espinosa (2003); y Lahiri y Ono (2000). En el primer trabajo citado, los autores analizan el caso de un mercado oligopólico donde compiten empresas nacionales e IED, determinando las cuotas óptimas a la emisión de contaminantes, el modelo es de equilibrio parcial para un bien homogéneo. Por su parte, Lahiri y Ono (2000); comparan dos instrumentos de política ambiental, cuotas e impuestos (un impuesto de contaminación es una cantidad que el gobierno cobra a las empresas por unidad de contaminación emitida - Cropper y Oates, 1992) para la producción de un bien homogéneo en presencia de IED, en competencia con empresas locales bajo condiciones de oligopolio, además de que considera que el país receptor es pequeño en comparación con el inversor.

De esta manera, el modelo desarrollado en este trabajo se distingue tanto del trabajo de Palomera y Espinosa (2003) como del estudio de Lahiri y Ono (2000), en el supuesto de la diferencia de tecnologías para abatir la contaminación entre empresas locales y firmas extranjeras de la IED. Palomera y Espinosa (2003) hacen un estudio de como la política ambiental afecta la inversión extranjera directa. Sin embargo, en este trabajo no se considera que el gobierno tenga la intención explicita de atraer inversión extranjera directa, solamente como la afecta. Además asumen que las empresas son homogéneas en términos de costos medioambientales, lo cual es sumamente irreal. En este trabajo, a diferencia de Palomera y Espinosa (2003), se considera que la diferenciación de costos medioambientales de las empresas es fundamental para considerar una política de atracción de inversión extranjera directa, y que además el gobierno tiene la intención de atraer inversión extranjera directa considerando siempre la externalidad de contaminación que esta inversión pueda traer a partir de esta heterogeneidad de costos medioambientales de las empresas.

Por otra parte, en el trabajo de Lahiri y Ono (2000), asumen una misma tecnología para abatir contaminación por parte de las empresas domésticas y foráneas, lo cual se aleja de la realidad en términos de diferencias tecnológicas entre empresas, y no permite establecer una más realista política medioambiental para la atracción de inversión extranjera directa. En este trabajo, a diferencia de Lahiri y Ono (2000), se considera la heterogeneidad de tecnología para abatir la contaminación entre empresas locales y foráneas, lo que da un mejor enfoque de política ya que permite generar resultados diferenciados que no existen en el caso de Lahiri y Ono (2000). Distinto al trabajo de Lahiri y Ono (2000), la política medioambiental óptima en este trabajo depende de esta capacidad de diferenciar las tecnologías para abatir contaminación de parte de las empresas domésticas y foráneas. 
El presente estudio relaciona la competencia entre empresas extranjeras y empresas nacionales con diferencias tecnológicas para abatir contaminación y las políticas ambientales. Determina además, la cuota de contaminación óptima como una política estratégica establecida por el gobierno anfitrión para alentar la entrada de empresas extranjeras, pero al mismo tiempo para contribuir a la conservación del medio ambiente, a través del control y la reducción de contaminantes.

La estructura del trabajo es la siguiente; inicia con la especificación y delimitación del modelo en la sección 2; en la sección 3 se realiza el análisis de estática comparativa; en la sección 4 se calcula la cuota óptima de contaminación. Por último, se establecen conclusiones en la sección 5.

\section{El modelo}

Siguiendo a Lahiri y Ono (2000), se considera un modelo de equilibrio parcial de una industria oligopólica en la que varias empresas nacionales y extranjeras, ubicadas en un país anfitrión, compiten en el mercado por un producto no comercializable. Las empresas extranjeras pueden ser consideradas como Inversión Extranjera Directa (IED) en el país anfitrión. Implícitamente, existe un bien numerario producido bajo condiciones de competencia perfecta, y existe sólo un factor de producción móvil, digamos la mano de obra, con rendimientos constantes a escala en todos los sectores. Por lo tanto, el salario se determina en el sector competitivo y los costos marginales en el sector oligopólico son constantes.

Se asume la existencia de $n$ empresas locales idénticas, y $m$ empresas extranjeras idénticas de la IED, donde $n$ y $m$ son enteros y $n \geq 1$ y $m \geq 1$. Cada empresa tiene una percepción basada en los supuestos de Cournot, es decir, presupone la producción de otras empresas como dada mientras maximiza su producción. ${ }^{2}$ Ambos tipos de empresas producen el mismo bien homogéneo y emiten cierta cantidad de contaminación en el proceso de producción. En este sentido, se asume que existe una externalidad negativa causada por esta contaminación. Estas firmas poseen una tecnología específica y diferenciada para disminuir la contaminación.

La estructura de costos entre $n$ firmas locales y entre $m$ firmas extranjeras es simétrica con respecto a su propio tipo. Por lo tanto, se puede asumir que la producción de cada empresa en el país anfitrión es la misma con respecto a su propio tipo. Los costos marginales de cada empresa son $k_{d}$ y $k_{f}$ para las empresas nacionales y extranjeras, respectivamente. Aquí y para el resto del documento, $d$ y $f$ son las notaciones para las empresas nacionales y extranjeras (sus componentes y políticas) ubicadas en el país anfitrión. Como se mencionó anteriormen-

\footnotetext{
${ }^{2}$ Los supuestos de Cournot para oligopolios consideran que existe un número constante de empresas idénticas que compiten (no cooperan) entre sí, producen sólo un bien homogéneo, tienen poder de mercado, eligiendo simultáneamente las cantidades producidas del bien al tiempo que maximizan sus beneficios.
} 
te, estos costos marginales se consideran constantes y, por lo tanto, equivalen a los costos variables promedio. Una parte de $k_{i}(i=d, f)$ está dada por la tecnología y las condiciones del mercado, y otra parte es inducida por la política ambiental, y se explicará más adelante.

El número de empresas nacionales es fijo, pero el número de empresas extranjeras, y por tanto la IED, puede verse afectado por el gobierno del país anfitrión con el uso de la política de control ambiental. El equilibrio de la IED se determina al equiparar las ganancias de cada empresa extranjera con un nivel de reserva dado exógenamente que las empresas extranjeras podrían obtener si se ubican en mercados alternativos.

En este modelo de equilibrio parcial, el producto homogéneo y no comerciable se produce bajo el esquema de oligopolio de Cournot mediante una función de demanda inversa y lineal como se describe a continuación,

$$
p=a-b Q
$$

Donde $p$ y $Q$ son respectivamente el precio y la demanda total bien homogéneo. Dado que el producto no es comerciable, la demanda interna total debe ser igual a la producción total, de modo que,

$$
Q=n x_{d}+m x_{f}
$$

Donde $x_{d} \mathrm{y} x_{f}$ representan la producción total de cada empresa nacional y extranjera, respectivamente. Considerando rendimientos constantes a escala y mercados perfectamente competitivos, se puede asumir que los costos marginales de las empresas nacionales y extranjeras, son constantes. Las ganancias de cada firma nacional y extranjera están dadas respectivamente por

$$
\begin{aligned}
& \pi_{d}=\left(p-k_{d}\right) x_{d} \\
& \pi_{f}=\left(p-k_{f}\right) x_{f}
\end{aligned}
$$

Se define $k_{i}$ como,

$$
k_{i}=c+T_{i}
$$

Donde $c$ es el costo tecnológico determinado por las condiciones del mercado, que se puede considerar constante, e idéntico tanto para las firmas locales como extranjeras. Como 
la producción de $x_{d}$ y $x_{f}$ implica la emisión de contaminación, $T i(i=d, f)$ es el costo unitario inducido por la política de reducción de la contaminación en cada empresa. Este costo inducido por la política ambiental se define como en Lahiri y Ono (2000) de la siguiente manera,

$$
T_{i}=\lambda_{i}\left(\theta-z_{i}\right)
$$

Donde $\theta$ es la cantidad de contaminación por unidad producida, de modo que $\theta \mathrm{x}_{d} \mathrm{y} \theta \mathrm{x}_{f}$ son la cantidad total de contaminación emitida por empresa (antes de la aplicación de la política ambiental) ubicada en el país anfitrión.3 Ambos tipos de empresas tienen la misma tecnología de producción y el nivel de contaminación que emiten es el mismo para todas las empresas. Sea $z_{i}(i=d, f)$, la cuota de contaminación por unidad producida que el gobierno anfitrión impone a las empresas. Por otro lado, la tecnología de reducción es tal que le cuesta a cada empresa una cantidad constante de $\lambda_{i}(i=d, f)$ disminuir una unidad de contaminación. Se asume que estos costos son diferentes entre los dos tipos de empresas. Se considera que las empresas extranjeras poseen una mejor tecnología para disminuir la contaminación de manera que

$$
\lambda_{d}>\lambda_{f}
$$

Este es el supuesto crucial que diferencia este trabajo y el de Lahiri y Ono (2000), y resulta mucho más realista sobretodo dentro de los países en desarrollo. Por otro lado, el número de empresas nacionales es fijo, mientras que el número de empresas extranjeras es endógeno. El gobierno puede afectar el número de empresas extranjeras cambiando los valores de la cuota de contaminación $z_{i}$. Se supone que el país receptor es pequeño en el mercado de IED. La empresa extranjera se muda o se va del país anfitrión si la ganancia que obtiene en el país anfitrión, $\pi f$, es más grande o más pequeña que la ganancia de reserva , que puede obtener en el resto del mundo. Por lo tanto, el equilibrio de la IED está dado por,

$$
\pi_{f}=\bar{\pi}
$$

Se supone que las empresas nacionales y extranjeras se comportan bajo los supuestos de Cournot-Nash. Cada empresa toma su decisión de producción tomando en cuenta los niveles de producción de otras empresas, el número de empresas y la cuota de contaminación establecida por el gobierno anfitrión. El equilibrio se define mediante un modelo de tres etapas: primero, el gobierno elige el nivel de cuotas diferenciadas tomando todo lo demás como dado; en la

\footnotetext{
${ }^{3}$ Implícitamente, este parámetro de contaminación unitaria, $\theta$, se considera superior al nivel que la Organización Mundial de la Salud (OMS) considera inofensivo.
} 
segunda etapa, el número de empresas extranjeras se determina dado el nivel de subsidios y niveles de producción; finalmente, se determinan los niveles de producción. Utilizando (3) y (4) se tienen las condiciones de primer orden que maximizan la utilidad de las empresas,

$$
\begin{aligned}
& b x_{d}=\left(p-k_{d}\right) \\
& b x_{f}=\left(p-k_{f}\right)
\end{aligned}
$$

Se obtiene la producción óptima de cada empresa a partir de (9) y (10) usando (1) - (4), tal que las soluciones óptimas son

$$
\begin{aligned}
& x_{d}=\frac{a+m k_{f}-(m+1) k_{d}}{\Delta b} \\
& x_{f}=\frac{a+n k_{d}-(n+1) k_{f}}{\Delta b}
\end{aligned}
$$

Donde

$$
\Delta=m+n+1
$$

Usando (11) y (12) en (3) y (4) resulta

$$
\begin{gathered}
\pi_{d}=b x_{d}^{2} \\
\pi_{f}=b x_{f}^{2}
\end{gathered}
$$

Las ecuaciones (1) - (6) y (11) - (14) forman la columna vertebral para el siguiente análisis. Ahora bien, se define el bienestar del país anfitrión teniendo en cuenta la aplicación de la cuota de contaminación. El bienestar del país anfitrión, $W$ puede ser escrito como,

$$
W=n \pi_{d}+m \pi_{f}+E C-\delta R
$$

Donde los primeros dos términos en (15) son los beneficios totales de empresas nacionales y extranjeras, el tercer término representa el excedente del consumidor y el último término se refiere a la desutilidad causada por la contaminación. ${ }^{4}$ Es bien conocido que el excedente del consumidor $E C$ se puede expresar así,

$$
E C=b Q^{2} / 2
$$

\footnotetext{
${ }^{4}$ En este documento solo consideramos que la contaminación daña el país donde se genera. Por lo tanto, se omite el análisis de la contaminación transfronteriza. Ver, por ejemplo, Copeland (1996), Copeland y Taylor (1995) y Hatzipanayotou, Lahiri y Michael (2002) para el análisis de la contaminación transfronteriza.
} 
En el último término de (15), $R$ es la cantidad total de contaminación emitida en el país receptor. Es la emisión bruta o la liberación total de contaminantes al aire, agua o tierra (por ejemplo, una tonelada de dióxido de azufre o de óxido nitroso); y la fuente de una externalidad negativa. $R$ se define como

$$
R=n z_{d} x_{d}+m z_{f} x_{f}
$$

Donde $z_{i}(i=d, f)$ es la cuota de contaminación por unidad producida que impone el gobierno a cada empresa ubicada en el país anfitrión, que es la misma para todas las empresas nacionales, y es la misma para todas las empresas extranjeras. Si las empresas no contaminaran $R$ sería cero. Finalmente, en el último término (15), $\delta$, es la desutilidad marginal por contaminar que se asume como constante, como lo hacen Lahiri y Ono (2000) y Markusen, Morey y Olewiler (1993 y 1995). ${ }^{5}$ En la siguiente sección, se determinarán algunos resultados de estática comparativa de la aplicación de las cuotas de contaminación como política de control ambiental.

\section{Estática comparativa}

Habiendo descrito las propiedades del modelo, se analizará la estática comparativa de la aplicación de la cuota de contaminación con respecto a las variables clave. Se considerará el efecto de una cuota de contaminación sobre la producción, el excedente del consumidor, el excedente del productor y la desutilidad de la contaminación. De (5) se obtiene

$$
\begin{gathered}
\frac{d k_{d}}{d z_{d}}=-\lambda_{d}>0 \\
\frac{d k_{f}}{d z_{f}}=-\lambda_{f}>0
\end{gathered}
$$

Un incremento en la cuota de contaminación de cualquiera de las dos empresas, reduce su costo de abatir la contaminación, y en consecuencia el costo marginal total. De (8), (12) y (14), utilizando derivación implícita resulta

$$
\begin{aligned}
\frac{d m}{d z_{d}}=-\frac{n \lambda_{d}}{b x_{f}}<0 \\
\frac{d m}{d z_{f}}=\frac{(n+1) \lambda_{f}}{b x_{f}}>0
\end{aligned}
$$

\footnotetext{
${ }^{5}$ Otros autores, como Asako (1979), consideran que la desutilidad marginal es una función creciente de la producción. Sin embargo, este supuesto alternativo no contradice nuestros resultados y una desutilidad marginal constante es un supuesto más conveniente.
} 
A mayor cuota de contaminación permitida a las empresas domésticas menor es el número de empresas foráneas entrantes, pues estas pierden competitividad ante las primeras. Contrariamente, mientras mayor es la cuota de contaminación permitida a las empresas foráneas mayor es el número de estas empresas que entran al país anfitrión, pues reducen sus costos de producción y ganan en competitividad. Utilizando (20) y (21) en (11) y (12) se obtiene

$$
\begin{gathered}
\frac{d x_{d}}{d z_{d}}=\frac{\lambda_{d}}{b}>0 \\
\frac{d x_{d}}{d z_{f}}=-\frac{\lambda_{f}}{b}<0
\end{gathered}
$$

Un aumento en la cuota de contaminación para las empresas domésticas reduce sus costos marginales de producción, lo cual incentiva su producción. Pero un aumento en la cuota de contaminación para las empresas foráneas reduce la competitividad de las empresas locales reduciendo su productividad.

$$
\frac{d x_{f}}{d z_{d}}=\frac{d x_{f}}{d z_{f}}=0
$$

Dada por la endogeneidad del número de empresas extranjeras, el cambio en las cuotas de contaminación no afecta la producción interna óptima de las empresas extranjeras. De (2), y tomando (22) - (24) se obtiene

$$
\begin{gathered}
\frac{d Q}{d z_{d}}=0 \\
\frac{d Q}{d z_{f}}=\frac{\lambda_{f}}{b}>0
\end{gathered}
$$

La producción total en el país anfitrión no se ve afectada por las variaciones en la cuota impuesta a las firmas locales porque la producción de la IED ajusta dichos cambios por la entrada o salida de firmas extranjeras, dependiendo de si la cuota sube o baja respectivamente. Por otro lado, la producción total se incrementa si la cuota de contaminación a las firmas extranjeras aumenta, ya que los costos marginales de dichas firmas disminuyen. Utilizando (25) y (26) en (16) resulta

$$
\begin{aligned}
& \frac{d E C}{d z_{d}}=0 \\
& \frac{d E C}{d z_{f}}=Q \lambda_{f}>0
\end{aligned}
$$


Debido a la endogeneidad de la IED el excedente del consumidor no cambia con la variación en una cuota a la contaminación en las empresas domésticas. Sin embargo, cuando la cuota de contaminación a las empresas extranjeras aumenta, el costo marginal de las empresas foráneas baja, entran más empresas extranjeras y aumenta el excedente del consumidor. De (13), (14) y (15), usando (22), (23) y (24) se obtiene

$$
\begin{gathered}
\frac{d\left(n \pi_{d}\right)}{d z_{d}}=2 n x_{d} \lambda_{d}>0 \\
\frac{d\left(n \pi_{d}\right)}{d z_{f}}=-2 n x_{d} \lambda_{f}<0
\end{gathered}
$$

Un aumento en la cuota de contaminación doméstica aumenta el excedente del productor de la empresa local porque disminuye el costo marginal de producción. Un aumento en la cuota de contaminación de la empresa foránea le proporciona una ventaja competitiva y reduce el excedente del productor de la empresa doméstica. Por parte del excedente del productor foráneo se tiene que

$$
\begin{gathered}
\frac{d\left(m \pi_{f}\right)}{d z_{d}}=-n x_{f} \lambda_{d}<0 \\
\frac{d\left(m \pi_{f}\right)}{d z_{f}}=(n+1) x_{f} \lambda_{f}>0
\end{gathered}
$$

De la misma manera un aumento en la cuota de contaminación doméstica disminuye el excedente del productor de las empresas foráneas porque les genera una desventaja competitiva. Contrariamente, un aumento en la cuota de contaminación foránea aumenta el excedente de los productores extranjeros porque disminuye su costo de producción. Finalmente de (17), y de (20) a (24) se obtiene

$$
\begin{gathered}
\frac{d R}{d z_{d}}=n x_{d}+\frac{n \lambda_{d}}{b}\left(z_{d}-z_{f}\right) \\
\frac{d R}{d z_{f}}=m x_{f}+\frac{\lambda_{f}}{b}\left(z_{f}(n+1)-z_{d} n\right)
\end{gathered}
$$

El efecto de la cuota de contaminación en la cantidad de contaminación total emitida es ambiguo. El cambio en la cantidad total de contaminación emitida por ambas empresas depende de la cantidad de contaminación emitida por cada tipo de empresa. Por lo tanto, el impacto de una cuota de contaminación sobre la desutilidad de la contaminación también es ambiguo. 


\section{Cuotas óptimas}

Una vez determinados los resultados de estática comparativa, se calculará la cuota de contaminación óptima siempre que se cumpla la condición de segundo orden. Derivando (15) con respecto a las cuotas de contaminación y usando de (27) - (34) resulta

$$
\begin{aligned}
d W= & {\left[2 n x_{d} \lambda_{d}-n x_{f} \lambda_{d}-\delta\left(n x_{d}+\frac{n \lambda_{d}}{b}\left(z_{d}-z_{f}\right)\right)\right] d z_{d} } \\
& +\left[-n x_{d} \lambda_{f}+\Delta x_{f} \lambda_{f}-\delta\left(m x_{f}+\frac{\lambda_{f}}{b}\left(z_{f}(n+1)-z_{d} n\right)\right)\right] d z_{f}
\end{aligned}
$$

La condición de concavidad requiere que la Condición de Segundo Orden (CSO) sea

$$
\operatorname{CSO}=\left(\frac{d^{2} W}{d z_{d}^{2}}\right)\left(\frac{d^{2} W}{d z_{f}^{2}}\right)-\left(\frac{d^{2} W}{d z_{d} d z_{f}}\right)\left(\frac{d^{2} W}{d z_{f} d z_{d}}\right)>0
$$

Ahora bien, la CSO también requiere que

$$
\begin{aligned}
& \frac{d^{2} W}{d z_{d}^{2}}=\frac{2 n \lambda_{d}}{b}\left(\lambda_{d}-\delta\right)<0 \\
& \frac{d^{2} W}{d z_{f}^{2}}=\frac{\lambda_{f}}{b}\left(\lambda_{f}(2 n+1)-\delta 2(n+1)\right)<0
\end{aligned}
$$

Lo cual se cumple si . Por otra parte

$$
\frac{d^{2} W}{d z_{d} d z_{f}}=\frac{d^{2} W}{d z_{f} d z_{d}}=-\frac{n}{b}\left[\lambda_{f} A_{1}+\lambda_{d} A_{2}\right]
$$

Donde

$$
\begin{aligned}
& A_{1}=\left(\lambda_{d}-\delta\right)<0 \\
& A_{2}=\left(\lambda_{f}-\delta\right)<0
\end{aligned}
$$

Y por definición

$$
A_{1}>A_{2}
$$

Sustituyendo (37), (38) y (39) en (36) y simplificando se obtiene

$$
\text { CSO }=\frac{n}{b}\left[2 n \lambda_{d} \lambda_{f} A_{1} A_{2}-2 \lambda_{d} \lambda_{f}^{2} A_{1}-n\left(\lambda_{d} A_{2}-\lambda_{f} A_{1}\right)\right]
$$


La CSO será positiva si $\delta>\lambda_{d}>\lambda_{f}$ Es decir, si la desutilidad marginal por contaminar es mayor que los costos de abatimiento, siempre se obtendrá una solución de esquina.

Igualando a cero los términos dentro de los corchetes en (35) y resolviendo para ambos niveles de cuotas de contaminación, resultan las cuotas óptimas que se dan a continuación,

$$
\begin{aligned}
& z_{d}^{*}=-\frac{b}{\lambda_{d} \lambda_{f} \delta}\left[\delta\left((n+1) \lambda_{f} x_{d}+m \lambda_{d} x_{f}\right)-\lambda_{d} \lambda_{f}\left((n+2) x_{d}+m x_{f}\right)\right] \\
& z_{f}^{*}=-\frac{b}{\lambda_{d} \lambda_{f} \delta}\left[\delta\left(n \lambda_{f} x_{d}+m \lambda_{d} x_{f}\right)-\lambda_{d} \lambda_{f}\left(n x_{d}+(m+1) x_{f}\right)\right]
\end{aligned}
$$

Considerando el cumplimiento de las condiciones de segundo orden, es fácil ver de (41) y (42) que si $\delta>>\lambda_{d}>\lambda_{f}$ entonces la política óptima será $z_{d}{ }^{*}=z_{f}{ }^{*}=0$. Lo cual se puede resumir en la siguiente proposición.

Proposición 1. En el equilibrio no cooperativo, si $\delta>>\lambda_{\mathrm{d}}>\lambda_{\mathrm{f}}$, las cuotas óptimas de contaminación son nulas $\left(z_{d}{ }^{*}=z_{f}{ }^{*}=0\right)$

En términos intuitivos, si la desutilidad marginal de contaminar es muy grande, entonces el gobierno aplica la mayor restricción posible, que consiste en exigir a las empresas que no contaminen en lo más mínimo, para reducir el impacto negativo de los daños de la contaminación en la salud de las personas. En este caso, ocurre un aumento en los costos marginales de producción, debido al incremento significativo en los costos por reducir la contaminación, en las empresas locales y extranjeras, que afecta negativamente al excedente del productor y del consumidor. Por otro lado, el beneficio dado por la reducción de la contaminación en la salud de las personas es mayor que la pérdida en el excedente del consumidor y del productor.

De (41) y (42), se observa también que cuando la desutilidad marginal de la contaminación se acerca por la derecha, y es cercana al costo de abatir la contaminación por parte de las empresas domésticas; y el costo de abatir contaminación por parte de las empresas foráneas se acerca por la izquierda y es cercano al costo por abatir contaminación de las empresas domésticas. Es decir, si $\delta \rightarrow \lambda_{d} \leftarrow \lambda_{f}$, se puede asumir, cumpliendo la CSO, que $\delta \approx \lambda_{\mathrm{d}} \approx \lambda_{\mathrm{f}}$, de modo que (41) y (42) se pueden reescribir como

$$
\begin{aligned}
& z_{d}^{*}=\frac{b x_{d}}{\lambda_{d}}>0 \\
& z_{f}^{*}=\frac{b x_{f}}{\lambda_{d}}>0
\end{aligned}
$$

Las dos fórmulas anteriores y el párrafo precedente se pueden enunciar en la siguiente proposición. 
Proposición 2. En el equilibrio no cooperativo, si $\delta \rightarrow \lambda_{d} \leftarrow \lambda_{d}$, las cuotas óptimas de contaminación son $\left(z_{d}^{*}>0\right.$ y $\left.z_{f}^{*}>0\right)$.

Intuitivamente, si el daño marginal causado por la contaminación no es significativo y, por concavidad, similar al costo de abatir la contaminación de las empresas domésticas y foráneas, el gobierno permite tanto a las empresas locales como extranjeras cierta cantidad de emisiones, lo que favorece las ganancias de las empresas y el excedente del consumidor; y aumenta el costo social por contaminar. En este caso, las cuotas de contaminación positivas representan un ahorro importante tanto para las empresas locales como para las extranjeras, ya que se reduce el costo marginal de producción. Ahora bien de (41) y (42) se obtiene

$$
z_{f}^{*}-z_{d}^{*}=\frac{b}{\lambda_{d} \delta}\left[\lambda_{d} x_{f}+x_{d}\left(\delta-2 \lambda_{d}\right)\right]>0
$$

Es claro ver que la expresión anterior es positiva bajo cualquiera de los dos casos arriba mencionados ya que por definición, dado que el costo de abatir contaminación es menor en la empresa foránea que la doméstica, entonces la producción de la empresa foránea es mayor a la de la empresa doméstica, y debido a esto se le permite una cuota mayor.

\section{Conclusiones}

La relación entre el medio ambiente y la IED es un problema que ha crecido rápidamente desde que la IED se ha convertido en un pilar para el desarrollo económico de las naciones y las preocupaciones ambientales han sido un tema candente en la política social. Las empresas extranjeras pueden ser ambientalmente más eficientes, pero pueden ser más contaminantes que las empresas nacionales. En este documento, se considera que las empresas extranjeras son técnicamente más eficientes para reducir la contaminación. En este sentido, el presente trabajo desarrolla un modelo de equilibrio parcial teórico bajo un marco de oligopolio en un país que recibe IED y posee inversión nacional para el mercado de un bien homogéneo, en el que también se supone que las empresas extranjeras tienen una tecnología más eficiente para disminuir la contaminación.

El gobierno anfitrión impone una cuota a la contaminación para maximizar el bienestar de sus ciudadanos. El gobierno anfitrión considera tanto el beneficio dado por las empresas extranjeras y nacionales, el excedente del consumidor, así como el daño causado por la contaminación en la salud de las personas. La determinación de la cuota de contaminación óptima contempla dos casos importantes en términos de la aplicación de la política ambiental que maximiza el bienestar en el país anfitrión.

En el primer caso, cuando la desutilidad marginal por contaminar es significativamente alta. En esta situación, siempre se establece una cuota cero a la contaminación, que incrementa 
ostensiblemente los costos de reducir la contaminación, y obviamente los costos marginales de producción, lo cual disminuye tanto el excedente del consumidor, así como la utilidad de las empresas locales como extranjeras. Por otro lado, la emisión de contaminantes en el ambiente se reduce sustancialmente lo cual reduce el costo social por contaminar.

En el segundo caso, cuando la desutilidad marginal para contaminar no es significativa, el gobierno decide aplicar una cuota de contaminación positiva, lo cual permite a las empresas emitir cierta cantidad de contaminantes, por lo tanto, se reducen los costos de abatimiento y los costos marginales de producción, lo cual favorece los beneficios de las empresas nacionales y extranjeras, y el excedente del consumidor. Aunque el aumento de las emisiones incremente el costo social por contaminar y afecte negativamente la salud de la población local.

No obstante, el modelo desarrollado es totalmente teórico y no está respaldado por evidencia empírica, aunque los autores trabajan en pruebas econométricas que validen los resultados obtenidos para un sector industrial de bienes homogéneos. El modelo puede extenderse en estudios futuros a mercados de bienes diferenciados; además se pueden establecer otros instrumentos de control medioambiental como impuestos de contaminación, permisos de contaminación transferibles o subsidios, y así poder determinar políticas óptimas específicas dependiendo del mecanismo de regulación ambiental elegido.

En conclusión, el modelo propuesto enfatiza la importancia del establecimiento racional de políticas ambientales estratégicas, que actúan en beneficio de todos los agentes económicos involucrados en el país receptor de IED, tanto las empresas locales como foráneas, los consumidores y el medio ambiente; al seleccionar aquellas políticas que también conducen al desarrollo sostenible de la economía, todos estos elementos están integrados en la función de bienestar general.

\section{Referencias}

Almeida, L. \& Rocha, S. (2009). Beyond Pollution Haloes: the Environmental Effects of FDI in the Pulp and Paper and Petrochemicals Sectors in Brazil. En K. Gallagher \& D. Chudnovsky (Eds.), Rethinking Foreign Investment for Sustainable Development: Lessons from Latin America. (pp. 201-216). Anthem Press. https://doi. org/10.7135/UPO9781843313243.012 Disponible en: https://pdfs.semanticscholar.org/7072/58ed4abba140fc573fbdc2b0c024185e2a72.pdf. Consultado: 06/06/2019.

Andonova, L. (2003). Openness and the Environment in Central and Eastern Europe: Can Trade and Foreign Investment Stimulate Better Environmental Management in Enterprises?. Journal of Environment and Development, 12(2), 177-204. https://doi.org/10.1177/1070496503012002003

Antweiler, W., Copeland, R. \& Taylor, S. (2001). Is Free Trade Good for the Environment?. The American Economic Review, 91(4), 877-908. https://doi.org/10.3386/w6707. Disponible en: https://www.jstor.org/stable/2677817. Consultado: 18/06/2019.

Asako, K. (1979). Environmental Pollution in an Open Economy. Economic Record, 55(4), 359-367. https://doi. org/10.1111/j.1475-4932.1979.tb02241.x 
Borensztein, E., Gregorio, J. \& Lee, W. (1998). How Does Foreign Direct Investment Affect Economic Growth?. Journal of International Economics, 45(1), 115-135. https://doi.org/10.1016/S0022-1996(97)00033-0

Cantwell, J. (1994). The Relationship Between International Trade and International Production. En D. Greenway y A. Winters (Eds.), Surveys in International Trade. (pp. 303-328). UK: Blackwell.

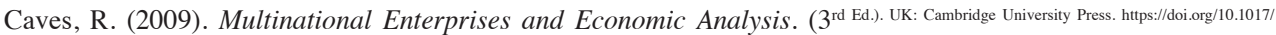
Св09780511619113

Chilchilnisky, G. (1994). North-South Trade and the Global Environment. American Economic Review, 84(4), 851874. Disponible en: https://www.jstor.org/stable/2118034. Consultado: 10/06/2019.

Cole, M., Elliot, R. \& Strobl, E. (2008). The EnvironmentalPerformance of Firms: The Role of Foreign Ownership, Training and Experience. Ecological Economics, 65(3), 538-546. https://doi.org/10.1016/j.ecolecon.2007.07.025. Disponible en: https://www.sciencedirect.com/science/article/pii/S0921800907004181. Consultado: 03/06/2019.

Copeland, R. (1996). Pollution Content Tariffs, Environmental Rent Shifting, and the Control of Crossborder Pollution. Journal of International Economics, 40(3-4), 459-476. https://doi.org/10.1016/0022-1996(95)01415-2

Copeland, R. \& Taylor, S. (1995). Trade and Transboundary Pollution. American Economic Review, 85(4), 716737. Disponible en : https://www.jstor.org/stable/2118228. Consultado : 14/06/2019.

Cropper, M. \& Oates W. (1992). Environmental Economics: A Survey. Journal of Economics Literature, 30(2), 675-740. Disponible en: https://www.jstor.org/stable/pdf/2727701.pdf. Consultado: 31/05/2019.

Eskeland, G. \& Harrison, A. (2003). Moving to Greener Pastures? Multinationals and The Pollution-Haven Hypothesis. Journal of Development Economics, 70(1), 1-23. https://doi.org/10.1016/S0304-3878(02)00084-6

Gentry, B. (1998). Private Capital Flows and the Environment: Lessons from Latin America. UK: Edward Elgar Cheltenham Publishing.

Hatzipanayotou, P., Lahiri, S. \& Michael, M. (2002). Can Cross-Border Pollution Reduce Pollution?. Canadian Journal of Economics, 35(4), 805-818. https://doi.org/10.1111/1540-5982.00155. Disponible en: https://www. jstor.org/stable/3131836. Consultado: 23/06/2019.

Haibo, C., Ayamba, E., Agyemang, A., Afriyie, S. \& Anaba, A. (2019). Economic Development and Environmental Sustainability- the Case of Foreign Direct Investment Effect on Environmental Pollution in China. Environmental Science and Pollution Research, 26(7), 7228-7242. https://doi.org/10.1007/s11356-019-04167-5

Kanbur, R., Keen, M. \& Wijnbergen, V. (1995). Industrial Competitiveness, Environmental Regulation, and Foreign Direct Investment. En The Economics of Sustainable Development. UK: Cambridge University Press.

Kolstad, C. (2012). Intermediate Environmental Economics. ( $2^{\text {nd Ed.). UK: Oxford University Press. }}$

Lahiri, S. \& Ono, Y. (2000). Protecting Environment in the Presence of Foreign Direct Investment: Tax Versus Quantity Restriction. Economics Discussion Papers 9985, University of Essex.

Li, Z., Dong, H., Huang, Z., \& Failler, P. (2019). Impact of Foreign Direct Investment on Environmental Performance. Sustainability, 11(13), 3538. https://doi.org/10.3390/su11133538. Disponible en: https://www.mdpi. com/2071-1050/11/13/3538/htm. Consultado: 01/06/2019.

Markusen, J., Morey, E. \& Olewiler, N. (1993). Environmental Policy when Market Structure and Plant Locations are Endogenous. Journal of Environmental Economics and Management, 24(1), 69-86. https://doi.org/10.1006/ jeem.1993.1005

Markusen, J., Morey, E. \& Olewiler, N. (1995). Competition in Regional Environmental Policies when Plant Locations are Endogenous. Journal of Public Economics, 56(1), 55-77. https://doi.org/10.1016/00472727(94)01419-O

Markusen, J. \& Venables, A. (1999). Foreign Direct Investment as a Catalyst for Industrial Development. European Economic Review, 43(2), 335-356. https://doi.org/10.1016/S0014-2921(98)00048-8

Motta, R. (2006). Analyzing the Environmental Performance of the Brazilian Industrial Sector. Ecological Economics, 57(2), 269-281. https://doi.org/10.1016/j.ecolecon.2005.04.008. Disponible en: https://www.sciencedirect.com/science/article/abs/pii/S0921800905002065. Consultado: 17/06/2019. 
Palomera, G. \& Espinosa, R. (2003). Políticas Ambientales en la Presencia de Inversión Extranjera Directa y Fusiones. Tesis de maestría, CUCEA, Universidad de Guadalajara.

Queiroz, F. (2018). La Formación de Paraísos de Contaminación: un Estudio de Caso de la Producción de Celulosa en el Cono Sur. EURE, 44(132) 213-237. https://doi.org/10.4067/s0250-71612018000200213. Disponible en: https://scielo.conicyt.cl/pdf/eure/v44n132/0250-7161-eure-44-132-0213.pdf. Consultado: 14/06/2019.

Silva, M. \& Madeira, J. (2015). Inversión Extranjera Directa y Medio Ambiente: Estado del Arte en la Investigación. Economía, 40(39), 11-36. Disponible en: https://www.redalyc.org/articulo.oa?id=195648436002. Consultado: $11 / 06 / 2019$.

United Nations Conference on Trade and Development (2017). Foreign Direct investment: Inward and Outward Flows and Stock, Annual. Disponible en: https://unctadstat.unctad.org/wds/TableViewer/tableView.aspx?ReportId=96740. Consultado: 13/06/2019.

United Nations Environment Programme (2018). Programme performance report 2018. Disponible en: https:// wedocs.unep.org/bitstream/handle/20.500.11822/27734/PPR_2018_FINAL.pdf. Consultado : 22/06/2019.

Xing, Y. \& Kolstad C. (2002). Do Lax Environmental Regulations Attract Foreign Investment?. Environmental and Resource Economics, 21, 1-22. https://doi.org/10.1023/A:1014537013353

Zarsky, L. (1997). Stuck In the Mud? Nation-States, Globalization and the Environment. Disponible en: http:// oldsite.nautilus.org/archives/papers/enviro/zarsky_mud.html. Consultado: 07/06/2019. 